Meta

Journal des traducteurs

Translators' Journal

\title{
Texte et paratexte dans la traduction assermentée des documents universitaires : une approche contrastive français-espagnol
}

\section{Gemma Andújar Moreno}

Volume 58, numéro 1, avril 2013

URI : https://id.erudit.org/iderudit/1023810ar

DOI : https://doi.org/10.7202/1023810ar

Aller au sommaire du numéro

Éditeur(s)

Les Presses de l’Université de Montréal

ISSN

0026-0452 (imprimé)

1492-1421 (numérique)

Découvrir la revue

Citer cet article

Andújar Moreno, G. (2013). Texte et paratexte dans la traduction assermentée des documents universitaires : une approche contrastive français-espagnol.

Meta, 58(1), 66-86. https://doi.org/10.7202/1023810ar
Résumé de l'article

À partir de l'étude contrastive d'un corpus de documents universitaires français et leurs traductions assermentées en espagnol, l'objectif du présent travail est d'analyser, d'un point de vue fonctionnaliste, les techniques de traduction appliquées dans le processus de transfert textuel. Grâce aux concepts de méthode de traduction et de technique de traduction, l'analyse contrastive permet d'examiner non seulement les aspects textuels de la traduction assermentée, mais aussi la dimension paratextuelle de cette modalité de traduction spécialisée. Les résultats mettent en évidence la tension entre adéquation et acceptabilité que sous-tend toujours le processus de traduction et le rôle essentiel que joue la culture de départ dans la détermination de la méthode de traduction globale. 


\title{
Texte et paratexte dans la traduction assermentée des documents universitaires: une approche contrastive français-espagnol
}

\author{
GEMMA ANDÚJAR MORENO* \\ Universitat Pompeu Fabra, Barcelone, Espagne \\ gemma.andujar@upf.edu
}

\begin{abstract}
RÉSUMÉ
À partir de l'étude contrastive d'un corpus de documents universitaires français et leurs traductions assermentées en espagnol, l'objectif du présent travail est d'analyser, d'un point de vue fonctionnaliste, les techniques de traduction appliquées dans le processus de transfert textuel. Grâce aux concepts de méthode de traduction et de technique de traduction, l'analyse contrastive permet d'examiner non seulement les aspects textuels de la traduction assermentée, mais aussi la dimension paratextuelle de cette modalité de traduction spécialisée. Les résultats mettent en évidence la tension entre adéquation et acceptabilité que sous-tend toujours le processus de traduction et le rôle essentiel que joue la culture de départ dans la détermination de la méthode de traduction globale.
\end{abstract}

\begin{abstract}
Starting from the contrastive study of a corpus of French academic transcripts and their corresponding sworn translations into Spanish, the present article aims to analyse the translation techniques that have been applied in the translation process from a functionalist perspective. By applying the concepts of translation technique and translation method, our contrastive analysis deals with textual as well as paratextual aspects of sworn translation. The results show the tension between adequacy and acceptability brought to bear by the translation process, as well as the important role played by the source culture in the determination of the global translation method.
\end{abstract}

\section{MOTS-CLÉS/KEYWORDS}

traduction assermentée français-espagnol, techniques de traduction, méthode de traduction, textes administratifs, documents universitaires

French-Spanish sworn translation, translation techniques, translation method, administrative texts, academic transcripts

\section{Introduction}

La traduction assermentée a été traditionnellement considérée comme une modalité de la traduction spécialisée très influencée par la culture de départ, dans le cadre de laquelle les experts traducteurs suivent une méthode de traduction "par défaut» consistant à rendre d'une façon littérale la forme et le contenu du texte de départ (TD) sans introduire d'explication sur leurs interprétations (Mayoral Asensio 2002: 12). Cette littéralité dominante a pour résultat des textes d'arrivée (TA) dans lesquels il est possible de distinguer la trace de la source à tous les niveaux textuels, parfois d'une façon peu vraisemblable pour la culture d'arrivée. Cette tendance de traduction est encouragée, et renforcée d'ailleurs, par l'exigence de «fidélité» à la culture de départ qu'impose le ministère des Affaires étrangères et de la Coopération (MAEC), 
organisme responsable des dispositions légales sur la validité des traductions officielles dans le domaine espagnol' ${ }^{1}$.

Par ailleurs, même si la publication des normes transitoires de l'Association Professionnelle Espagnole des Traducteurs et des Interprètes (APETI) ${ }^{2}$, l'une des premières tentatives rigoureuses d'unification méthodologique dans le domaine espagnol, date de 1992, on continue de reprocher aux experts traducteurs le manque d'homogénéité méthodologique. Toutefois, cette hétérogénéité méthodologique ne semble pas être une caractéristique exclusive du domaine espagnol:

If we search for a "norm" describing the way official translation is conducted around the world, we will not find it; the reality is too rich and varied. The models or prototypes that we come across are diverse and do not find exactly in any scheme. (Mayoral Asensio 2003: 4)

L'objectif du présent travail est de mieux cerner les spécificités du rapport unissant le texte de départ au texte d'arrivée dans le processus de traduction assermentée, à partir de l'étude contrastive d'un corpus de documents universitaires français et de leur traduction assermentée en espagnol. L'étude de cas que nous présentons a pour objet l'examen des ressources expressives dont l'expert traducteur dispose pour accomplir la commande de traduction, grâce à la description des techniques de traduction qui ont été appliquées dans le processus de transfert textuel et paratextuel.

L'opacité qui pèse sur les pratiques professionnelles des experts traducteurs et l'isolement relatif des membres de cette profession (Monzó Nebot 2003; Way 2005), qui se montrent dans la plupart des cas peu disposés à soumettre leurs traductions à l'analyse des chercheurs en traductologie, font de l'étude contrastive de corpus authentiques une source de données particulièrement utile pour y déceler des modèles de comportement permettant de mieux connaître le rôle des experts traducteurs comme médiateurs interculturels.

\section{Cadre théorique, corpus et méthodologie de l'étude}

La notion déjà classique de technique de traduction s'avère encore aujourd'hui un outil d'analyse déterminant dans les études descriptives qui visent à dégager des régularités dans les tendances de traduction. Les premières tentatives de systématisation des ressources expressives des traducteurs datent d'une époque où la traduction dépendait étroitement de la linguistique, marquée par les taxonomies de Vinay et Darbelnet (1958), Fedorov (1968) ou Levy (1969). Parmi ces premiers travaux, celui ayant eu le plus grand retentissement est sans doute la Stylistique comparée du français et de l'anglais de Vinay et Darbelnet (1958), même s'il a aussi été critiqué pour n'avoir pas pris en compte les coordonnées communicatives, pragmatiques et cognitives inhérentes au processus de traduction. Mais ces critiques n’ont pas empêché ces contributions de fournir le point de départ à d'autres réflexions plus actuelles ayant pour but d'élargir le champ des études traductologiques descriptives. Ainsi, la taxonomie des techniques de traduction proposée par Molina et Hurtado (2002), dans une perspective d'analyse fonctionnaliste, essaie de systématiser les classifications existantes élaborées à partir de critères hétérogènes. L'approche systématique de l'étude a permis de l'appliquer avec succès à la description de différentes modalités de la traduction, même si, à notre connaissance, cette taxonomie n'a pas encore été mise à l'épreuve dans le champ de la traduction assermentée ${ }^{3}$. 
Molina et Hurtado (2002) proposent la définition suivante, pour techniques de traduction:

[...] we define translation techniques as procedures to analyse and classify how translation equivalence works. They have five basic characteristics:

1) They affect the result of the translation

2) They are classified by comparison with the original

3) They affect micro-units of text

4) They are by nature discursive and contextual

5) They are functional.

(Molina et Hurtado 2002: 509)

L'application de ce concept à l'analyse contrastive du corpus devrait nous aider à mettre en lumière l'orientation générale du processus de traduction assermentée et d'en dégager les priorités. En outre, déceler les tendances principales des techniques de traduction permettrait aussi de formuler des hypothèses sur la méthode de traduction, l'option globale qui imprègne l'ensemble du texte, repérable à partir de la présence de certaines techniques et dépendante de la fonction de la traduction dans la culture d'arrivée.

Dans ce but, nous avons constitué un corpus textuel en appliquant deux critères généraux de sélection, qui répondent à la volonté de réunir un ensemble homogène et représentatif de traductions assermentées réelles, adéquat pour une analyse traductologique descriptive.

TABLEAU 1

\section{Critères de sélection du corpus textuel}

\begin{tabular}{|l|}
\hline Critères invariables \\
\hline Homogénéité temporelle \\
Homogénéité de la commande de traduction \\
Homogénéité générique \\
Homogénéité thématique \\
\hline Critères variables \\
\hline Nombre de documents retenus \\
Mécanismes textuels et paratextuels analysés \\
\hline
\end{tabular}

Ainsi, le corpus analysé est constitué de trente documents universitaires français et de leur traduction officielle en espagnol, faite par des professionnels espagnols entre les années 2001 et 2007 (homogénéité temporelle). Ces échantillons textuels s'inscrivent tous dans la même situation de communication (homogénéité de la commande de traduction) : il s'agit de documents délivrés par des universités françaises et appartenant tous à des étudiants francophones qui en ont présenté les traductions assermentées au secrétariat d'une même université espagnole comme l'une des conditions exigées pour y suivre des études. Le destinataire final de la traduction assermentée est donc l'administration de l'université espagnole, institution qui détermine tacitement les conditions d'acceptabilité du produit final. L'analyse de productions textuelles réelles répondant à une commande de traduction spécifique, et non de textes originaux s'inscrivant dans une commande hypothétique qui devrait être inférée par le chercheur, permet de préciser la fonction de la traduction assermentée dans la culture d'arrivée et d'évaluer avec une plus grande objectivité la pertinence des techniques de traduction appliquées. 
La morphologie textuelle du corpus est déterminée par l'appartenance des échantillons textuels à trois genres discursifs (homogénéité générique): diplôme universitaire, relevé de notes et attestation ${ }^{4}$. Ces genres, à leur tour, pourraient être classés dans la catégorie plus générale de «textes administratifs non normatifs d'attestation» [notre traduction] selon la classification du Ministerio para las Administraciones Públicas espagnol ${ }^{5}$, car ils peuvent être définis comme des documents dont le but est d'attester un acte, un fait ou des effets universitaires. Leur fonction pragmatique principale est donc informative: il s'agit de documents délivrés à la demande d'un étudiant souhaitant attester certaines informations de nature académique, mais ils sont aussi revêtus de la force illocutoire que leur confère l'institution émettrice et ont à ce titre des conséquences importantes sur des processus administratifs du domaine académique universitaire.

Le choix de textes du domaine académique (homogénéité thématique), et non d'autres documents qui pourraient aussi faire l'objet d'une traduction assermentée, est justifié par leur récurrence en tant que commande de traduction assermentée. En effet, comme l'affirment de nombreux auteurs (Mayoral Asensio 1991: 1; Márquez Villegas 2005: 34; Way 1997; 2005, pour n'en citer que quelques-uns) et comme le démontre aussi la pratique professionnelle, ce type de documents constitue l'une des commandes les plus fréquentes dans le champ de la traduction officielle grâce au nombre croissant d'étudiants qui souhaitent poursuivre des études dans un pays étranger.

Lapplication des critères exposés ci-dessus nous a permis de réunir un corpus de trente documents et de leur traduction. Dans le but d'analyser la configuration discursive de ce corpus, nous avons confronté des micro-segments textuels et paratextuels constitués d'un extrait du TD et de sa traduction correspondante. Ces microsegments, qui ont été choisis en raison de leur représentativité, présentent une longueur variable pouvant aller d'un terme actualisé dans un seul énoncé jusqu'à plusieurs énoncés organisés en paragraphes, dans le cas des phénomènes macrotextuels. En raison de la nature différente des mécanismes textuels et paratextuels, qui implique un traitement différent de la part de l'expert traducteur, nous avons examiné ces deux types de mécanismes d'une façon indépendante, en respectant leurs caractéristiques particulières.

\section{Analyse contrastive du corpus}

\subsection{Dimension textuelle}

Dans un premier temps, nous avons analysé l'agencement textuel des traductions assermentées, c'est-à-dire l'organisation macrotextuelle des documents conformément au genre textuel auquel ils appartiennent, pour examiner ensuite, au niveau microtextuel, la terminologie représentative du champ thématique, notamment la dénomination des diplômes et des matières, ainsi que l'expression des mentions universitaires.

\subsubsection{Organisation macrotextuelle}

La notion de genre discursif occupe toujours une place importante dans le champ de la traductologie, à en juger par les nombreuses études portant sur la problématique 
que posent le concept et son application à l'analyse contrastive (García Izquierdo 2007; Monzó Nebot 2001; 2002; 2003). Ce qui est indéniable, en tout cas, c'est le rapport étroit existant entre traduction et genre discursif. En effet, dans la perspective de la traduction en tant que processus, les genres discursifs véhiculent des instructions interprétatives générales orientant l'acte interprétatif du traducteur vers la construction d'un sens spécifique. Par ailleurs, si nous considérons la traduction en tant que produit textuel, une bonne connaissance des spécificités des genres discursifs dans les deux cultures de travail permettra à l'expert traducteur de produire un TA conforme aux attentes du nouveau destinataire. Enfin, il est essentiel de noter que les conventions génériques sont des indices culturels pouvant varier d'une culture administrative à une autre. L'appartenance du TD à un genre discursif donné exercera donc une influence notable sur les techniques de traduction appliquées.

Les diplômes, attestations et relevés de notes de notre corpus constituent une « colonie des genres» (Bhatia 2004: 57-84), qui partagent le même but communicatif, les mêmes conventions rhétoriques et présentent aussi des caractéristiques lexicogrammaticales identiques. Le comportement général des experts traducteurs du corpus consiste à reproduire, avec une fidélité scrupuleuse, l'organisation macrotextuelle des documents, notamment dans le cas du relevé de notes. En effet, ce genre du discours administratif qui semble répondre à des conventions textuelles assez souples dans les deux cultures administratives permet d'imiter sans grande difficulté l'agencement textuel du TD avec un résultat acceptable pour la culture d'arrivée.

Or, les conventions génériques des documents espagnols ne coïncident pas toujours avec celles des documents français. Des divergences macrotextuelles ont été détectées, surtout dans le cas des attestations françaises, dont l'organisation textuelle est à peu près identique à celle des documents espagnols. Le français en effet privilégie la rédaction à la première personne du singulier, tandis que le texte espagnol est rédigé à la troisième personne. Dans les traductions assermentées du corpus, cette convention n'est pas respectée, comme le montre l'exemple suivant:

(1) a. Je soussigné, directeur de la scolarité de l'Institut d'Études Politiques de Paris, certifie que Mademoiselle XXX, née le XXX à XXX, ayant subi les épreuves prévues par le règlement, par décision du Jury en date XXX, a obtenu le diplôme de l'Institut d'Études Politiques de Paris avec la mention Cum Laude.

(Attestation de l'Institut d'Études Politiques de Paris [DOC06_FR])

(1) b. Yo, el infraescrito, director de escolaridad del Instituto de Estudios Políticos de París, certifico que la Señorita XXX, nacida el XXX en XXX, tras superar las pruebas establecidas por el ordenamiento, y por decisión del jurado en fecha de XXXX, ha obtenido el Diploma del Instituto de Estudio Políticos de París con la calificación de cum laude.

(Attestation de l'Institut d'Études Politiques de Paris [DOC06_ES], traduction assermentée)

Le résultat de la traduction littérale est un document qui peut sembler peu naturel, mais sans doute pas incompréhensible, pour la culture administrative d'arrivée. Le fait que les traducteurs aient décidé majoritairement d'importer la formulation macrostructurale du TD est l'indice que la méthode de traduction globale met au premier plan l'adéquation en soulignant la fidélité formelle et sémantique moyennant la transposition des éléments référentiels. Par contre, des constituants textuels comme 
les unités phraséologiques n'ont pas été traduits littéralement, mais selon la technique que Molina et Hurtado (2002) appellent équivalent établi: «Established equivalent. To use a term or expression recognized (by dictionaries or language in use) as an equivalent in the TL [...]» (Molina et Hurtado 2002: 510). Cette technique est étroitement liée au genre discursif du TD:

(2) a. Cette attestation est établie pour servir et valoir ce que de droit.

(Attestation de l'Université Paris 7 - Denis Diderot ([DOC15_FR], souligné par l'auteur)

(2) b. Se expide la presente certificación para que conste y surta los efectos oportunos.

(Attestation de l'Université Paris 7 - Denis Diderot [DOC15_ES], traduction assermentée, souligné par l'auteur)

Ce comportement traducteur, majoritaire dans les attestations et les diplômes du corpus, est le recours expressif des experts traducteurs pour atténuer en quelque sorte l'effet d'étrangeté que pourrait provoquer un texte avec une organisation macrotextuelle peu naturelle dans la culture administrative d'arrivée. En conséquence, le résultat est un TA plus vraisemblable pour la culture cible, et donc plus acceptable.

\subsubsection{Niveau microtextuel: terminologie représentative du champ thématique}

L'implantation de l'Espace Européen d'Éducation Supérieure (EEES) dans l'Union Européenne a eu pour conséquence une nouvelle structuration du système universitaire espagnol et la création d'une nouvelle nomenclature (grades universitaires, crédits ECTS, masters...) en harmonie avec les universités européennes, ce qui facilite beaucoup la tâche de l'expert traducteur en ce qui a trait à la terminologie spécialisée. La période envisagée pour le corpus (2001-2007) a déterminé la sélection des termes analysés sous cette rubrique. Nous avons donc examiné les termes spécialisés appartenant à trois domaines spécifiques: la dénomination des diplômes universitaires, la dénomination des matières académiques et l'expression des mentions académiques. Ces termes, qui pourraient être considérés des culturèmes (Nord 1997: 34), offrent une information qui remplit la fonction pragmatique du document et qui devient, par là même, l'information la plus pertinente du point de vue de la commande de traduction assermentée.

\subsubsection{Dénomination des diplômes universitaires}

Les techniques de traduction appliquées pour rendre ces termes dans la langue d'arrivée constituent un reflet des différentes attitudes de l'expert traducteur à l'égard des culturèmes et du poids attribué à chacune des cultures administratives en jeu dans le processus de traduction. L'analyse contrastive révèle une progression entre le respect de la spécificité du culturème moyennant l'importation de formulations propres à la culture de départ et l'adaptation au terme le plus usité dans la culture d'arrivée ou le plus proche de celle-ci. Aux deux extrêmes de la gradation, des techniques de traduction pures ont été appliquées, tandis qu'aux niveaux intermédiaires les traducteurs ont choisi des techniques mixtes, comme l'illustre ce schéma: 
FIGURE 1

Échelle des techniques de traduction des culturèmes administratifs dans le corpus

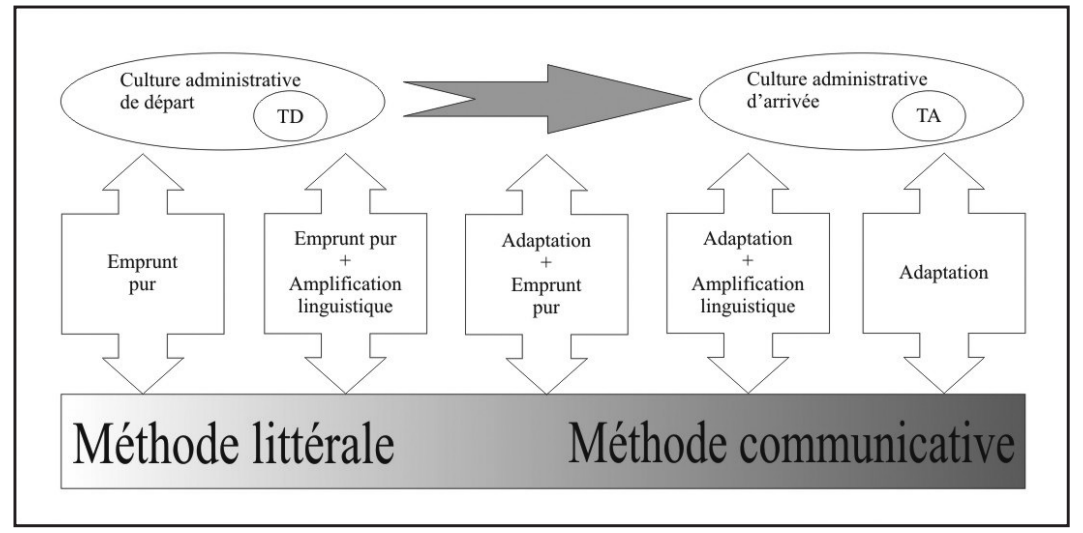

Au niveau de l'échelle le plus proche de la culture de départ, les traducteurs ont préféré l'emprunt dans sa modalité pure: «Borrowing. To take a word or expression straight from another language. It can be pure (without any change) [...] or it can be naturalized (to fit the spelling rules in the TL)» (Molina et Hurtado 2002: 510). Dans les micro-segments analysés, le terme est donc importé d'une façon directe (en italique), peut-être parce que les traducteurs ont estimé que les équivalents de la culture cible n'étaient pas tout à fait adéquats:

(3) a. DEUG 2 MCC (Relations Sociales)

(Relevé de notes et résultats de l'Université de Nice-Sophia Antipolis [DOC04_FR])

(3) b. DEUG 2 MCC (Relaciones Sociales)

(Relevé de notes et résultats de l'Université de Nice-Sophia Antipolis [DOC04_ES], traduction assermentée)

L'avantage de cette technique est qu'elle permet de garder la spécificité dénominative du diplôme, même si, comme le montre l'exemple ci-dessus, l'acronyme DEUG (Diplôme d’Études Universitaires Générales), sans que n'y soit ajoutée une explication de la part du traducteur, peut s'avérer peu clair pour les destinataires de la traduction assermentée qui ne possèdent pas de solides connaissances du système universitaire français.

Au deuxième niveau, la technique de l'emprunt a été appliquée en combinaison avec l'amplification linguistique, moyennant l'insertion d'une note en bas de page. Selon Molina et Hurtado (2002: 510), l'amplification peut être définie en ces termes: "Amplification. To introduce details that are not formulated in the ST: information, explicative paraphrasing [...]. Footnotes are a type of amplification [...]».

Le micro-segment suivant en est un exemple: 
(4) a. Maîtrise d'Arts Plastiques

(Diplôme de l'Université de Paris 8 [DOC26_FR])

(4) b. Maîtrise de Artes Plásticas

[...]

i. Nota del traductor: La maîtrise es el grado universitario francés que se consigue al finalizar el segundo ciclo de una determinada carrera universitaria y que equivaldría a la «licenciatura»

[i. Note du traducteur: La maîtrise est le grade universitaire français validant un deuxième cycle de l'enseignement supérieur. Ce diplôme est équivalent à une «licenciatura» espagnole]

(Diplôme de l'Université de Paris 8[DOC26_ES], traduction assermentée; notre rétrotraduction)

Le traducteur choisit dans ce cas-là l'importation directe du terme français, mais il y ajoute un éclaircissement au moyen de données qu'il suppose interprétables par le destinataire, afin de faciliter la compréhension et d'ainsi garantir l'efficacité de la traduction assermentée. Ces notes en bas de page sont intéressantes pour l'analyse dans la mesure où elles explicitent les connaissances encyclopédiques que l'expert traducteur considère indispensables pour le destinataire. Même si cette technique est très minoritaire dans le corpus étudié, il s'agit de l'option recommandée par des auteurs comme Way (1997) ou Mayoral Asensio (1999b; 2003) ${ }^{6}$.

Les traducteurs s'approchent un peu plus de la culture administrative d'arrivée quand ils combinent les techniques d'adaptation et d'emprunt entre parenthèses:

(5) a. Maîtrise de Sciences Économiques

(Diplôme de l'Université Strasbourg I [DOC01_FR])

(5) b. Licenciatura (Maîtrise) en Ciencias Económicas

(Diplôme de l'Université Strasbourg I [DOC01_ES], traduction assermentée)

Molina et Hurtado définissent l'adaptation comme suit: «To replace a ST cultural element with one from the target culture [...]» (2002: 509). Ce procédé implique le recours au culturème le plus proche de la langue cible; une dénomination qui, même si elle n'est pas exacte dans la plupart des micro-segments analysés, offre l'avantage de la familiarité pour le destinataire. En outre, l'inclusion du culturème français entre parenthèses, donc en position hiérarchique secondaire, semble répondre à une volonté de signaler que la maîtrise n'a pas tout à fait les mêmes caractéristiques académiques que la licenciatura du système universitaire espagnol, comme c'est le cas dans l'exemple ci-dessus.

Quand les experts traducteurs appliquent en même temps les techniques d'adaptation et d'amplification, ils rendent explicites dans la traduction des nuances qui sont absentes du TD:

(6) a. Maîtrise de Sciences de Gestion

(Diplôme de l'Université Paris I-Panthéon-Sorbonne [DOC09_FR])

(6) b. Licenciatura con tesina de Ciencias de Gestión

(Diplôme de l'Université Paris I-Panthéon-Sorbonne [DOC09_ES], traduction assermentée) 
Dans cette traduction, par exemple, l'introduction du complément con tesina (avec mémoire) semble répondre à la même intention: compenser, bien que de façon imparfaite, les différences sémantiques entre les deux culturèmes.

Au niveau de la gradation le plus proche de la culture d'arrivée, l'adaptation dans sa modalité pure est la technique employée majoritairement dans le corpus. Ce microsegment nous en offre un exemple:

(7) a. Licence de Sciences Économiques

(Relevé de notes de l'Université Louis Pasteur [DOC14_FR])

(7) b. Diplomatura en Ciencias Económicas

(Relevé de notes de l'Université Louis Pasteur [DOC14_ES], traduction assermentée)

Il s'agit d'une traduction qui cherche à établir l'équivalence administrative, centrée sur la culture administrative d'arrivée et qui implique une adaptation imparfaite. En effet, selon la fonction de la traduction assermentée dans la culture d'arrivée, cette divergence peut poser des problèmes d'acceptabilité, puisque les deux concepts en jeu ne sont pas du tout coïncidents et interchangeables?.

L'analyse du corpus a donc révélé que, en ce qui concerne des culturèmes comme les dénominations des diplômes, la priorité est accordée à l'acceptabilité pour la culture d'arrivée au détriment de l'adéquation à la culture de départ, car les techniques de traduction qui cherchent à établir des équivalences administratives sont les plus fréquentes. Cependant, il faut souligner le large éventail de techniques de traduction mixtes (et donc plus créatives) employées par les traducteurs afin d'établir un pont interculturel entre les deux systèmes d'éducation supérieure; ces traductions sont des formulations plus élaborées engageant une plus grande visibilité de l'expert traducteur.

\subsubsection{Dénomination des matières}

L'équivalent établi par le dictionnaire est la technique appliquée dans tous les cas du corpus pour rendre les dénominations des matières:

(8) a. Muséologie et restauration

(Relevé de notes de l'Université de Nantes [DOC23_FR])

(8) b. Museología y restauración

(Relevé de notes de l'Université de Nantes [DOC23_ES], traduction assermentée)

(9) a. Méthodes d'analyse financière

(Relevé de notes de l'Université Louis Pasteur [DOC24_FR])

(9) b. Métodos de análisis financiero

(Relevé de notes de l'Université Louis Pasteur [DOC24_ES], traduction assermentée)

Étant donné que les matières d'une formation universitaire sont aussi des culturèmes du système éducatif au même titre que la dénomination des diplômes, le traitement tout à fait différent qu'elles ont mérité dans le corpus peut surprendre. En effet, les matières ne se prêtent pas aux techniques de traduction mixtes que nous avons observées dans le cas des diplômes, puisqu'elles ont toujours été rendues mot à mot. 


\subsubsection{Expression des mentions académiques}

Certains auteurs comme Mayoral Asensio (1991; 1999b) ou Way (1997) soulignent la complexité de la traduction des mentions académiques en raison de son caractère culturel, au point de la considérer comme l'un des écueils plus importants de la traduction officielle. Dans le corpus, la majorité des erreurs de traduction relèvent de ce domaine. L'analyse contrastive a mis en lumière deux cas de figure différents: quand les notes s'expriment sous forme numérique sur dix ou vingt points, la tendance généralisée est de respecter le même choix dans les traductions:

(10) a. Théorie des organisations Note: 4,50 sur 10

(Attestation de l'Université Paris 7 - Denis Diderot [DOC15_FR])

(10) b. Teoría de las organizaciones Nota: 4,50 sobre 10

(Attestation de l'Université Paris 7 - Denis Diderot [DOC15_ES], traduction assermentée)

Par contre, quand les notes s'expriment sous la forme d'une mention, les traducteurs privilégient toujours l'adaptation au système culturel d'arrivée, comme l'illustre cet exemple:

(11) a. Toutes les notes sont exprimées sur 20. L’obtention d'une moyenne générale (en gras) supérieure ou égale à 10 assortie d'une mention Passable, Assez-Bien, Bien ou Très-Bien vaut l'acquisition de tous les modules.

(Relevé de notes de l'Université Louis Pasteur [DOC20_FR])

(11) b. Todas las notas están expresadas sobre 20. La obtención de una media general (en negrita) superior o igual a 10 acompañada de una mención Suficiente, Bien, Notable o Sobresaliente confiere la aprobación de todos los módulos.

(Relevé de notes de l'Université Louis Pasteur [DOC20_ES], traduction assermentée)

Les traducteurs choisissent en général la formulation la plus habituelle dans le système académique espagnol, même si dans celui-ci les notes ne sont pas calculées de la même manière. Cette tendance, illustrée par le micro-segment ci-dessus, est confirmée par d'autres traductions relevées dans le corpus. Pourtant, Catherine Way (2005) déconseille en ces termes le type de traduction cherchant ainsi l'équivalence administrative: «It is not part of the sworn translator's mission to recognize a given mark for another, so the use of dynamic equivalents is virtually impossible unless required explicitly by the client» (Way 2005: 182). L'établissement des équivalences administratives serait donc une tâche qui dépasserait les attributions professionnelles de l'expert traducteur. Le recours à l'emprunt combiné à l'amplification linguistique est la solution proposée pour éviter ce type de traduction, même si cette technique mixte représente encore un choix très minoritaire:

(12) a. [...] la Maîtrise Arts Plastiques, mention Très bien, est décernée à [...]

(Diplôme de l'Université Paris 8 [DOC26_FR]) 
(12) b. Se le expide la Maîtrise de Artes Plásticas, calificación "très bien"ii a [...] ii. N. del T.: En el sistema francés se puntúa sobre 20 y el aprobado se obtiene con una nota igual o superior a 10. La calificación "très bien" se obtiene normalmente con una nota superior a 16 y equivaldría en el sistema español a "sobresaliente".

[ii. N.d.T. Dans le système de notation français, les étudiants sont notés sur 20 et l'obtention d'une note égale ou supérieure à 10 permet de réussir. La mention «très bien» est accordée normalement aux notes supérieures à 16. Dans le système espagnol, cette mention est équivalente à sobresaliente.]

(Diplôme de l'Université Paris 8 [DOC26_ES], traduction assermentée; notre rétrotraduction)

Dans l'exemple ci-dessus, le traducteur explique les différences entre les deux systèmes académiques pour justifier une éventuelle adaptation de la note qui soit compréhensible pour le destinataire espagnol. Par contre, dans d'autres micro-segments du corpus, l'amplification ne comporte pas l'explicitation des adaptations espagnoles éventuelles, mais consiste plutôt en une traduction littérale des sigles français sous la forme d'une note du traducteur incorporée au texte principal:

(13) a. P/ P / B /AB / TB / P /AB

(Relevé de notes de l'Université Louis Pasteur [DOC20_FR])

(13) b. P / P / B /AB / TB / P /AB

N. T. $\mathrm{P}=$ Suficiente, $\mathrm{AB}=$ Bastante Bien, $\mathrm{B}=$ Bien $\mathrm{TB}=$ Muy Bien

(Relevé de notes de l'Université Louis Pasteur [DOC20_ES], traduction assermentée)

Cet exemple tiré d'un relevé de notes de l'Université Louis Pasteur permet d'observer trois erreurs dans la traduction de « $\mathrm{AB}$ » (Assez Bien, 12-13/20), « $\mathrm{B} »(B i e n$, 16-17/20) y «TB» (Très Bien, 18-20/20), qui entravent la compréhension du destinataire et faussent la note obtenue pour le titulaire du diplôme. D'abord, dans le système de notation espagnol, la mention bastante bien n'existe pas et on accorde la mention aprobado aux étudiants ayant obtenu un résultat dans cet intervalle. Ensuite, la mention française «bien» équivaut approximativement au notable espagnol (7-8/10) et, enfin, la note la plus élevée de l'échelle de notation espagnole est sobresaliente et pas muy bien. Avec la reformulation mot à mot, le traducteur assermenté tombe dans le piège du faux ami ne reconnaissant pas qu'il a affaire à un culturème. La mauvaise interprétation de la mention constitue un autre problème fréquent, comme le montre l'exemple suivant:

(14) a. La Maîtrise de Sciences de Gestion, mention Assez Bien, est décernée à [...]

(Diplôme de l'Université Paris I-Panthéon-Sorbonne [DOC09_FR])

(14) b. La licenciatura en Gestión, calificación notable, es otorgada a [...]

(Diplôme de l'Université Paris I-Panthéon-Sorbonne [DOC09_ES], traduction assermentée)

L'erreur de traduction, qui semble s'expliquer, dans le cas présent, par une connaissance insuffisante du système de notes français, n'est pas sans conséquences. En effet, cette équivalence erronée se manifeste par l'attribution, dans la traduction assermentée, d'une note supérieure à la note réelle (Assez Bien, 12-13/20 et Notable, 
7-8/10), ce qui revêt une gravité particulière, puisqu'il s'agit d'une traduction certifiée ayant une valeur officielle et pouvant donc influer directement sur les processus administratifs dans la culture d'arrivée.

\subsection{Dimension paratextuelle}

Les textes de notre corpus contiennent aussi des éléments paratextuels de type verbal et iconique, qui sont placés apparemment hors de l'espace textuel, mais qui dépendent étroitement de celui-ci, et qui ont la même importance pour la commande de traduction assermentée que la traduction interlinguistique. La typologie des techniques de traduction interlinguistique s'est avérée très utile pour déceler les tendances de traduction de ce type d'éléments, même s'il a fallu introduire quelques modifications dans le but d'améliorer l'analyse contrastive.

\subsection{1 Éléments paratextuels relevant du genre discursif des documents}

\subsubsection{Signatures}

Les documents académiques contiennent tous la signature du titulaire, ainsi que celles des responsables académiques et administratifs qui certifient leur validité. Ces signatures sont toujours accompagnées du nom et du prénom du signataire en caractères d'imprimerie. Pour signaler l'apparition d'une signature dans les TA, les experts traducteurs optent pour ce que Molina et Hurtado appellent la description: «Description. To replace a term or expression with a description of its form and/or function» (2002: 510). Toutefois, dans ce type de traduction, la signature, un élément graphique, est remplacée par sa description linguistique; c'est pourquoi nous avons appelé cette technique de traduction description intersémiotique, car il ne s'agit pas d'un transfert entre deux langues, mais bien d'une adaptation d'un élément graphique au milieu linguistique.

Comme nous le verrons plus loin, la description intersémiotique constitue la technique la plus utilisée pour rendre en espagnol les éléments paratextuels des documents académiques. Les données du corpus permettent d'observer un large éventail de variantes formelles, dont la plus fréquente est l'introduction de la description dans le texte sans aucune marque linguistique permettant de distinguer la voix du traducteur de celle du TD (exemples 15 et 16). Les exemples suivants montrent la voix du traducteur commentant la signature (firma):

(15) Firma

[Signature]

(Relevé de notes de l'Université Louis Pasteur [DOC20_ES], traduction assermentée; notre rétrotraduction)

(16) Firma ilegible

[Signature illisible]

(Attestation d'unités de valeur de l'Université Paris 7 [DOC02_ES], traduction assermentée; notre rétrotraduction)

(17) Firmado por B. A.

[Signé par B. A.]

(Attestation de l'Université Paris 7 - Denis Diderot [DOC15_ES], traduction assermentée; notre rétrotraduction) 
(18) Fdo: C. B. [Signé (en abrégé) : C. B.]

Ces solutions de traduction configurent un texte polyphonique hybride, dans lequel deux voix distinctes sont situées au même niveau. D’autres micro-segments, comme (17) ou (18), font apparaître l'identité du signataire (Signé par...; Signé [en abrégé]: ...), solution apparemment peu recommandable, puisqu'elle engage la responsabilité personnelle de l'expert traducteur ${ }^{8}$. L'intervention de celui-ci, et la visibilité qui en résulte, va encore un peu plus loin dans les exemples suivants:

(19) Firmado: Auzanneau

(Attestation de l'Institut d'Études Politiques de Paris [DOC06_ES], traduction assermentée)

(20) La titular: No consta firma

(Diplôme de l'Université de Nantes [DOC22_ES], traduction assermentée)

Dans l'exemple (19), le traducteur transcrit une signature lisible en italique pour souligner son caractère manuscrit ; à l'opposé, dans le micro-segment (20), le traducteur choisit de ne pas reproduire le manque d'information du TD, mais plutôt de fournir une information métatextuelle à propos d'une signature qui d'après lui devrait figurer dans le document (La titulaire: Pas de signature).

La tendance secondaire, en ce qui concerne la visibilité du traducteur, consiste à signaler graphiquement l'intervention de celui-ci au moyen de parenthèses ou de crochets, comme le montrent ces exemples:

(21) (Firma)

[(Signature)]

(Diplôme de l'Université de Nantes [DOC22_ES], traduction assermentée; notre rétrotraduction)

(22) (Firmado)

[(Signé)]

(Diplôme de l'Université Paris I-Panthéon-Sorbonne [DOC09_ES], traduction assermentée; notre rétrotraduction)

(23) [Firma ilegible]

[[Signature illisible]]

(Diplôme de l'Université de Nantes [DOC22_ES], traduction assermentée; notre rétrotraduction)

(24) [Firma del rector del distrito universitario]

[[Signature du Recteur de l'académie]]

(Diplôme de l'Université de Nantes [DOC22_ES], traduction assermentée; notre rétrotraduction)

Comme on peut le constater, la typographie est le recours expressif dont le traducteur dispose pour distinguer son commentaire du contenu du TA. Les exemples ci-dessus montrent aussi une progression très nette dans l'intervention du traducteur. En effet, dans (21) et (22), le traducteur se limite à signaler l'apparition d'une signature; dans (23), la description est plus précise, car le traducteur y ajoute une carac- 
téristique de celle-ci (illisible) que le TD ne formule pas explicitement; tandis que, finalement, le traducteur affirme en (24) que le signataire est le responsable académique mentionné (recteur du district universitaire). Cette dernière solution engage, de nouveau, l'expert traducteur quant à l'identité du signataire.

\subsubsection{Tampons}

Les tampons des institutions et des universités délivrant les documents académiques visent à certifier leur authenticité et à leur conférer validité légale. Le corpus analysé montre trente occurrences de tampons de formes différentes. Pour traduire ces éléments graphiques, la technique la plus récurrente est de type mixte: description intersémiotique combinée à la traduction (équivalent établi par le dictionnaire) ou à la reproduction des éléments linguistiques inclus à intérieur du tampon. Une fois de plus, le corpus révèle un éventail de variantes formelles, mais l'option prédominante consiste à inclure explicitement la voix du traducteur au même niveau hiérarchique que celle du texte de départ. Les micro-segments (25) et (26) en sont des exemples:

(25) Sigue un sello redondo que reza: Universidad de Nantes, Ap. de Correos 13522, 44035 Nantes Cedex

[Il y a un sceau rond qui indique: Université de Nantes, BP 13522, 44035 Nantes Cedex]

(Diplôme de l'Université de Nantes [DOC22_ES], traduction assermentée; notre rétrotraduction)

(26) Sello: Universidad Louis Pasteur - Estrasburgo - UFR Ciencias Económicas y de Gestión

[Sceau: Université Louis Pasteur - Strasbourg - UFR des Sciences Économiques et de Gestion]

(Relevé de notes de l'Université Louis Pasteur [DOC08_ES], traduction assermentée; notre rétrotraduction)

(27) [Sello] Universidad Louis Pasteur

[[Sceau] Université Louis Pasteur]

(Relevé de notes de l'Université Louis Pasteur [DOC24_ES], traduction assermentée; notre rétrotraduction)

Dans l'exemple (27), par contre, des crochets délimitant l'intervention du traducteur placent celle-ci dans une position secondaire. Cette technique n'est appliquée que dans deux cas parmi une trentaine d'exemples analysés; il s'agit donc d'une solution peu utilisée, comme c'était le cas dans la catégorie des signatures. En outre, dans (26) et (27) le traducteur n'a pas recours à un verbe introducteur, tandis que dans (25) il a opté pour une formule plus élaborée. La plupart des traductions analysées ne rendent pas explicite, par les descriptions intersémiotiques, la forme du tampon (circulaire, octogonale...), même lorsque la présence de plusieurs tampons différents pourrait induire le destinataire en erreur. Cette tendance à la synthèse dans la description des tampons se manifeste d'une façon extrême dans le cas suivant:

(28) (Sello de la dirección del centro)

[(Sceau de la direction du centre)]

(Certificat de l'Institut d'Études Politiques de Paris [DOC07_ES], traduction assermentée; notre rétrotraduction) 
Dans ce dernier exemple tiré d'un certificat, la formule Institut d'Études Politiques de Paris - Direction du tampon original a été traduite par une expression plus hyperonymique (sello de la dirección del centro) appartenant à la catégorie généralisation telle que la définissent Molina et Hurtado: "Generalization : to use a more general or neutral term» (2002: 510).

Les tampons et les signatures sont des éléments graphiques étroitement liés, à un point tel qu'ils se superposent dans certains documents académiques. Dans ces cas, le traducteur profite d'une seule et même description intersémiotique pour décrire simultanément ces deux types d'éléments, comme nous avons pu l'observer à maintes reprises:

(29) [Figura una firma ilegible junto con el sello de la Universidad de París VIII] [[Signature illisible à côté du tampon de l’Université de Paris VIII]]

(Attestation de résultats de l'Université Paris 8 [DOC19_ES], traduction assermentée; notre rétrotraduction)

Quand les tampons contiennent des informations complètement illisibles, ce fait est signalé dans le TA; quand l'information est partiellement illisible, les traducteurs optent pour indiquer l'extrait illisible et traduire ou décrire le reste des données:

(30) Sello illegible

(Relevé de notes et résultats de l'Université de Nice-Sophia Antipolis [DOC05_ES], traduction assermentée)

(31) Sello: (Ileg.) Ciencias Humanas

(Relevé de notes et résultats de l'Université de Nice-Sophia Antipolis [DOC04_ES], traduction assermentée)

Les données du corpus montrent donc que la plupart des traducteurs assermentés respectent la consigne énoncée notamment par Mayoral Asensio, quand il affirme qu'il est nécessaire d'indiquer dans les traductions officielles les rectifications, les additions, la cassure du papier ou les fragments incomplets du texte de départ (1999b: 68-69). Cependant, cette information n'apparaît pas entre parenthèses ou entre crochets, comme le conseillent les spécialistes (Mayoral Asensio 1999b; Duro Moreno 2013).

Enfin, il convient de souligner que, dans huit des trente occurrences analysées, la traduction espagnole ne contient aucune référence à la présence d'un ou de plusieurs tampons dans le TD, même s'il s'agit d'éléments essentiels sans lesquels le document n'aurait pas de validité officielle.

\subsubsection{Certification conforme}

Sept des trente documents français sont certifiés conformes à l'original, avec un cachet faisant foi ajouté a posteriori. Ces certifications constituent la marque d'un acte administratif par lequel un responsable public autorisé certifie l'équivalence totale entre un document et une photocopie, sans cependant se prononcer sur l'authenticité du document original. La technique de traduction appliquée dans tous les cas est la traduction littérale, mot à mot, celle-ci ne prenant pas en considération les unités phraséologiques de la langue d'arrivée: 
(32) a. Vu, Collationné et Certifié Conforme à l'original qui nous a été présenté. Paris, le 28 juin 2002

Pour le Maire et par délégation Le Fonctionnaire Municipal Délégué

(Attestation d'unités de valeur de l'Université Paris 7 [DOC02_FR])

(32) b. Visto, cotejado y certificado fiel al original que nos ha sido presentado.- París, 28 de junio de 2002.- Por el Alcalde de París y por delegación, el funcionario municipal delegado.

(Attestation d'unités de valeur de l'Université Paris 7 [DOC02_ES], traduction assermentée)

Il découle de cette technique que le traducteur a estimé acceptable d'importer dans la traduction les spécificités structurelles et formelles du TD, comme le triplet $v u$, collationné et certifié conforme ou le recours à la voix passive, même si cette littéralité entraîne un texte peu naturel pour la culture d'arrivée. Une solution plus adéquate, sans doute, serait d'adopter la formulation phraséologique du système administratif d'arrivée, qui est beaucoup plus simple: Compulsado y conforme con el original. Ce type d'incursion dans la culture d'arrivée exige la recherche et l'exploitation de textes parallèles du même genre discursif de la part de l'expert traducteur.

La certification conforme non seulement atteste l'équivalence entre le document original et la photocopie, mais offre aussi de l'information sur le processus de traduction assermentée en signalant que le document a été traduit à partir d'une photocopie; aussi est-il important d'indiquer son apparition dans la traduction. Cependant, dans trois des sept cas analysés les traducteurs omettent toutes les références aux certifications conformes. Une fois de plus, le corpus montre le peu d'importance que les traducteurs ont accordé à un élément paratextuel ayant une fonction très précise dans le processus administratif.

\subsubsection{Blasons et logotypes}

La plupart des documents administratifs étudiés ont un en-tête avec le logotype ou le blason de l'institution émettrice, qui apparaît généralement accompagné du nom de l'institution et de son adresse postale. Ainsi, logotype et texte constituent non seulement une unité indivisible mais aussi la marque d'identité de l'institution; cette unité transmet de l'information sur le plan sémiotique, car elle véhicule des inférences sur le poids de l'institution dans le domaine académique de départ tout en officialisant le document.

La technique pour rendre les blasons et les logotypes dans les traductions est, dans tous les cas, ce que nous avons appelé omission partielle: les traducteurs n'indiquent pas l'apparition d'un élément graphique, mais ils reproduisent les éléments linguistiques (nom de l'institution et adresse postale), même quand ceux-ci se trouvent inclus dans le logotype. L'élément graphique est donc négligé par les traducteurs, malgré sa valeur sémiotique.

\subsubsection{D’autres éléments graphiques}

Certains textes du corpus présentent des ratures, comme celle que nous reproduisons ci-dessous:

(33) Ayant soutenu avec succès un travail d'études et des recherches sur le thème suivant:

(Attestation de diplôme de l'Université Paris 7 [DOC11_FR]) 
ou des inscriptions manuscrites adressées à l'institution de destination de l'étudiant, ajoutées a posteriori sur le document français:

(34) À destination d'Universidad Virtual de Empresa

(Attestation d'unités de valeur de l'Université Paris 7 [DOC02_FR])

(35) Document destiné aux autorités espagnoles

(Attestation de l'Université Paris 7 - Denis Diderot [DOC15_FR])

(36) Document destiné aux autorités de l'Espagne

Attestation de réussite de l'Université Paris-Dauphine ([DOC16_FR])

Une fois de plus, les traducteurs choisissent de supprimer ces éléments graphiques, soit par négligence, soit parce qu'ils considèrent que l'information véhiculée est peu pertinente pour le destinataire de la traduction.

\subsection{2 Éléments paratextuels relevant de la méthodologie de la traduction assermentée}

Les éléments paratextuels englobés sous cette rubrique sont étroitement liés à la fonction qu'a l'expert traducteur de certifier la véracité de la traduction; aussi, ils sont clairement définis dans les dispositions du Ministère des Affaires étrangères et de la Coopération (MAEC) relatives à cette modalité de la traduction ${ }^{9}$. Dans son étude de 2003, Mayoral Asensio inclut ce type de paratextualité dans ce qu'il appelle les «conditions de succès de la traduction assermentée» (2003: 41), conditions qui, si elles ne sont pas satisfaites, pourraient entraîner l'annulation de l'acte de traduction en tant que tel.

\subsubsection{Formule de certification}

Toutes les traductions du corpus sont assorties de la formule de certification établie par le MAEC (BOE du 23 février 1996) en bas de page, sans aucune marque graphique la distinguant du texte principal. Cette formule est indispensable pour que la traduction assermentée soit considérée comme telle et ait une valeur officielle:

(37) X, intérprete jurado de francés, certifica que la que antecede es traducción fiel y completa al español de un documento original redactado en francés.

[X, expert traducteur en langue française, certifie que la traduction espagnole précédente est fidèle et conforme au document original rédigé en français.]

(Diplôme de l'Université Strasbourg I [DOC01_ES], traduction assermentée)

Il convient de souligner aussi que, dans deux cas, la formule de certification est modifiée: le genre discursif du document est mentionné d'une façon explicite avec l'expression "un título académico redactado en lengua francesa ${ }^{10}$ » (un document universitaire rédigé en langue française), où le terme générique documento (document) est remplacé par título (diplôme). En outre, les experts traducteurs dont nous avons analysé les traductions ne précisent pas dans la formule de certification s'ils ont traduit à partir d'une photocopie certifiée conforme, même s'il s'agit de ce type de document dans sept des trente textes analysés. 


\subsubsection{Tampon et signature de l'expert traducteur}

Les dispositions légales du MAEC n'exigent pas que les traductions assermentées portent le tampon et la signature du traducteur sur toutes les pages du texte, mais la plupart des professionnels suivent cette tendance. Par contre, ils n'ont pas recours au "visé», une signature en abrégé que l'expert traducteur peut utiliser sur toutes les pages du document, sauf la dernière.

\subsubsection{Support de la traduction assermentée}

Les dispositions du MAEC n'exigent pas non plus que la traduction assermentée soit faite sur papier timbré, mais les normes transitoires de l'Association Professionnelle Espagnole des Traducteurs et des Interprètes (APETI) ${ }^{11}$ et les auteurs comme Márquez Villegas $(1996$; 2005 : 25) en recommandent l'utilisation. Par contre, d'autres spécialistes comme Duro Moreno (2013) déconseillent le recours au papier timbré, étant donné que ce type de support augmente le prix du produit final, son coût étant à la charge du client. Cependant, l'analyse du corpus montre que le papier timbré n'est pas tombé en désuétude, à en juger par le nombre de traductions du corpus délivrées sur tel support, soit neuf des trente textes analysés. Le choix majoritaire demeure malgré tout le papier DIN A4; lorsque celui-ci est employé, il est fréquent de trouver des en-têtes avec le logotype du traducteur.

\section{Conclusions}

Notre analyse traductologique de trente traductions assermentées, même si ces traductions ont été effectuées dans le cadre d'une commande de traduction concrète avec des caractéristiques spécifiques, a mis en lumière le rôle de l'expert traducteur comme médiateur entre deux cultures administratives.

Tout d'abord, l'étude des ressources expressives des experts traducteurs a montré que la méthodologie de cette modalité de traduction est toujours très hétérogène dans le domaine espagnol. Nos conclusions coïncident aussi avec celles de Mayoral Asensio (1999b: 70), pour qui la traduction littérale est la méthode la plus souvent employée, tant pour la forme que pour le contenu. On peut donc affirmer que les traducteurs s'efforcent de rendre tous les éléments de la langue d'origine, négligeant pour ce faire les aspects discursifs des documents de départ.

Dans la dimension textuelle, la méthode de traduction littérale se concrétise par des techniques telles que l'emprunt, la traduction littérale ou l'équivalent établi par les dictionnaires, qui soulignent l'importance accordée à la culture de départ dans le processus global de traduction. Le recours à ces techniques a pour résultat des traductions assermentées dans lesquelles transparaît le texte source aux niveaux microtextuel et (surtout) macrotextuel d'une façon peu naturelle pour le destinataire final.

Cependant, il faut souligner aussi l'application ponctuelle de techniques de traduction orientées vers la culture d'arrivée, comme l'adaptation. Dans ces cas, la traduction qui cherche à établir des équivalences administratives ne résout pas toujours le manque de correspondance sémantique totale ou partielle entre les culturèmes du domaine académique. Entre ces deux extrêmes, nous trouvons une échelle de techniques de traduction mixtes (emprunt + amplification, adaptation + emprunt, adaptation + amplification) répondant au souci de conserver un équilibre difficile 
entre l'exigence de fidélité à la culture de départ imposée par le MAEC espagnol et l'exigence de précision et d'intelligibilité pour la culture d'arrivée imposée par la commande même.

Cet éventail de techniques de traduction mixtes mérite d'être mis en relief, car il reflète aussi la volonté de l'expert traducteur de construire des ponts interculturels précis à l'aide de ressources expressives plus créatives, même si cela implique un degré plus élevé d'intervention et de visibilité de sa part. Ces techniques de traduction, secondaires par leur fréquence d'application, pourraient s'inscrire dans le cadre d'une méthode de traduction interprétative-communicative, où les traducteurs tentent de conserver la finalité du texte original, sa fonction et son genre textuel, pour produire sur le destinataire final un effet administratif équivalent.

Dans notre étude, nous avons examiné les aspects interlinguistiques de la traduction assermentée sans négliger sa dimension paratextuelle. En effet, nous avons estimé important d'inclure cette dimension dans l'analyse contrastive, car elle pose des problèmes traductologiques spécifiques dont il faut tenir compte pour effectuer une description précise des tendances en matière de traduction. En ce sens, la taxonomie des techniques de traduction interlinguistique ne s'est avérée opérationnelle pour la traduction intersémiotique que grâce à l'introduction de certaines modifications. Ainsi, la technique la plus fréquente pour rendre les éléments paratextuels est celle que nous avons appelée «description intersémiotique», où l'élément graphique ou iconique du TD est remplacé par une description linguistique. C'est dans cette dimension paratextuelle que l'on peut observer le plus grand nombre d'omissions, d'erreurs et de négligences de la part des experts traducteurs, même si cette circonstance n'a pas entravé l'acceptabilité des traductions.

Le but de notre réflexion était de mettre en évidence la spécificité de la traduction assermentée, une modalité de la traduction spécialisée où les traducteurs doivent résoudre des problèmes de nature différente posés par la combinaison d'éléments textuels et paratextuels. Les techniques de traduction adoptées contribuent à souligner la nature hybride des documents académiques étudiés. En effet, les traductions assermentées analysées constituent un transgenre (Monzó Nebot 2002) avec des caractéristiques discursives propres, qui ne relèvent ni de la combinaison des conventions génériques de la culture administrative de départ ni de celles de la culture administrative d'arrivée. C'est pourquoi nous pouvons affirmer que ces textes oscillent, avec plus ou moins de succès, entre deux cultures linguistiques et administratives différentes.

\section{REMERCIEMENTS}

Nous tenons à remercier les évaluateurs anonymes, dont les remarques critiques ont permis d'améliorer le présent travail.

\section{NOTES}

* Étude réalisée dans le cadre du groupe de recherche CEDIT (Centre de Estudis de Discurs i Traducció), référence 2009 SGR 00771, Agència de Gestió d’Ajuts Universitaris i de Recerca, Generalitat de Catalunya.

1. Le Bureau d'Interprétation des Langues (Oficina de Interpretación de Lenguas) du Ministère des Affaires Étrangères et de la Coopération (MAEC) est chargé de nommer les experts traducteurs dans le domaine espagnol. La formule de certification exigée par cet organisme (BOE du 23 février 
1996) impose d'une façon explicite la fidélité au texte de départ: «Don / Doña (nombre y apellidos), Intérprete Jurado de (idioma), certifica que la que antecede es traducción fiel y completa al (lengua de destino) de un documento redactado en (lengua de origen). En (lugar), a (fecha)» [Traduction: M / Mme (prénom et nom), expert traducteur en (langue de départ), certifie que la traduction vers le (langue d'arrivée) précédent est fidèle et conforme à un document rédigé en (langue de départ). Lieu et date]. V. Boletín Oficial del Estado (23 de febrero 1996) : Orden de 8 de febrero de 1996 por la que se dictan normas sobre los exámenes para nombramiento de Intérpretes Jurados. Madrid: Boletín Oficial del Estado, 6877-6878.

2. Asociación Profesional Española de Traductores e Intérpretes (1992): Normas transitorias para el ejercicio profesional de los intérpretes jurados. Madrid: APETI.

3. Voir, par exemple, l'application de la taxonomie de Molina et Hurtado à la traduction journalistique (Hernández Guerrero 2006) ou à la traduction audiovisuelle (Martí Ferriol 2005).

4. Nous avons analysé six échantillons du genre diplôme universitaire, douze échantillons du genre relevé de notes et douze échantillons du genre attestation. Étant donné que la quantité de documents retenus est relativement petite, le traitement des données textuelles et paratextuelles a été réalisé de façon manuelle.

5. Ministerio para las Administraciones Públicas (1993): Manual de estilo del lenguaje administrativo. Madrid: MAP, 89-90.

6. Catherine Way (1997: 18), par exemple, suggère d'inclure dans les notes explicatives la durée, le niveau et le contenu des études en tant qu'information essentielle pour faciliter la compréhension du culturème et ainsi garantir l'efficacité communicative de la traduction officielle.

7. Il convient de signaler que la notion d'acceptabilité dans la commande de traduction analysée est assez souple: le fonctionnaire de l'université chargé de réviser la traduction officielle vérifie seulement que la traduction porte le tampon de l'expert traducteur, que celui-ci soit habilité par le MAEC et que les programmes des matières, tamponnés par l'université d'origine, soient inclus dans les documents produits. Ainsi, toute la responsabilité relative au contenu de la traduction incombe directement à l'expert traducteur. Teresa JuANOLA (19 juin 2009): communication personnelle, Barcelone.

8. Voir à cet égard les exemples dans Mayoral Asensio (1999a: 28).

9. Voir à cet égard Boletín Oficial del Estado (23 de febrero 1996) : Orden de 8 de febrero de 1996 por la que se dictan normas sobre los exámenes para nombramiento de Intérpretes Jurados. Madrid: Boletín Oficial del Estado, 6876-6878.

10. Attestation d'unités de valeur de l'Université Paris 7, traduction assermentée.

11. Asociación Profesional Española de Traductores e Intérpretes (1992): Normas transitorias para el ejercicio profesional de los intérpretes jurados. Madrid: APETI.

\section{RÉFÉRENCES}

BнатіA, Vijay K. (2004): World of Written Discourse. A Genre-based View. Londres/New York: Continuum.

Duro Moreno, Miguel (2013): La traducción jurada: propuesta de normalización estilística francés-español / español-francés. Tools4Translation. Consulté le 22 avril 2013, <http:// www.tools4translation.com/wp-content/uploads/2013/04/duro9.pdf $>$.

Fedorov, Andrei (1968): Introduction à la théorie de la traduction. (Traduit par Trad. École Supérieure de Traducteurs et d'Interprètes) Bruxelles: ESIT.

García Izquierdo, Isabel (2007): Los géneros y las lenguas de especialidad. In: Enrique Alcaraz VAró, dir. Las lenguas profesionales y académicas. Barcelona/Alicante: Ariel Lenguas Modernas-IULMA, 119-125.

Hernández Guerrero, María José (2006): Técnicas específicas de traducción periodística. Quaderns. Revista de Traducció. 13:125-139.

Levy, Jiri (1969): Die literarische Übersetzung: Theorie einer Kunstgattung. Frankfurt am Main: Athenäum.

MARtí Ferriol, José Luis (2005) : Estudio descriptivo y comparativo de las normas de traducción en las versiones doblada y subtitulada al español del filme Monsters' Ball. Puentes. Hacia nuevas investigaciones en la mediación intercultural. 6:45-52. 
Márquez Villegas, Luís (1996): Orientaciones metodológicas para la realización de traducciones juradas. In: Pedro San Ginés Aguilar et Emilio Ortega Arjonilla, dir. Introducción a la traducción jurídica y jurada francés-español. Granada: Comares, 95-101.

MÁrQUeZ VILLEgAS, Luís (2005): Formulario de traducciones juradas francés-español y españolfrancés. Granada: CSV.

Mayoral Asensio, Roberto (1991): La traducción jurada de documentos académicos norteamericanos. Gitrad. Web del Traductor Jurídic. Consulté le 13 décembre 2008, <http://www. gitrad.uji.es/common/articles/Mayoral5.pdf>.

Mayoral Asensio, Roberto (1999a): Las fidelidades del traductor jurado : una batalla indecisa. In: Manuel C. Feria García, dir. Traducir para la justicia. Granada: Comares, 17-57.

Mayoral Asensio, Roberto (1999b) : Traducción oficial (jurada) y función. In: Manuel C. Feria García, dir. Traducir para la justicia. Granada: Comares, 59-86.

Mayoral Asensio, Roberto (2002): ¿Cómo se hace la traducción jurídica? Puentes. Hacia nuevas investigaciones en la mediación intercultural. 2:9-14.

Mayoral Asensio, Roberto (2003): Translating Official Documents. Manchester: St. Jerome.

Molina, Lucía et Hurtado, Amparo (2002): Translation Techniques Revisited: A Dynamic and Functionalist Approach. Meta. 47(4):498-512.

Monzó Nевот, Esther (2001): El género discursivo: un concepto clave en la enculturación del traductor. In: Anne Barr, Jesús Torres del Rey et M. Rosario Martín Ruano, dir. Últimas corrientes teóricas en los estudios de traducción y sus aplicaciones. Salamanca: Ediciones de la Universidad de Salamanca, 486-500.

Monzó Nевот, Esther (2002): La professió del traducció jurídic i jurat: descripció sociològica del professional $i$ anàlisi discursiva del transgènere. Thèse de doctorat. Castelló: Universitat Jaume I. Consulté le 20 août 2011, <http://www.tdx.cat/handle/10803/10563>.

Monzó Nевот, Esther (2003): La traducción jurídica a través de los géneros: el transgénero y la socialización del traductor en los procesos de enseñanza/aprendizaje. Discursos. Revista de tradução. 2:21-36.

Nord, Christiane (1997): Translating as a Purposeful Activity. Functionalist Approaches Explained. Manchester: St. Jerome.

Vinay, Jean-Paul et Darbelnet, Jean (1958): Stylistique comparée du français et de l'anglais. Méthode de traduction. Paris: Didier.

WaY, Catherine (1997): The Translation of Spanish Academic Transcripts: Implications for Recognition. In: Karl Simms, dir. Translating Sensitive Texts. Linguistic Aspects. Amsterdam: Rodopi, 177-185.

WAY, Catherine (2005): Investigando la traducción como acción social: los documentos académicos español-inglés. In: Esther Monzó Nebot et Anabel Borja Albi, dir. La traducción y la interpretación en las relaciones jurídicas internacionales. Castelló: Universitat Jaume I, 255-265. 